\title{
Injectable Self-Healing Antibacterial Bioactive Polypeptide- Based Hybrid Nanosystems for Efficiently Treating Multidrug Resistant Infection, Skin-Tumor Therapy, and Enhancing Wound Healing
}

\author{
Li Zhou, Yuewei Xi, Yumeng Xue, Min Wang, Yanle Liu, Yi Guo, and Bo Lei*
}

The surgical procedure in skin-tumor therapy usually results in cutaneous defects, and multidrug-resistant bacterial infection could cause chronic wounds. Here, for the first time, an injectable self-healing antibacterial bioactive polypeptide-based hybrid nanosystem is developed for treating multidrug resistant infection, skin-tumor therapy, and wound healing. The multifunctional hydrogel is successfully prepared through incorporating monodispersed polydopamine functionalized bioactive glass nanoparticles (BGN@ PDA) into an antibacterial F127- $\varepsilon$-Poly-L-lysine hydrogel. The nanocomposites hydrogel displays excellent self-healing and injectable ability, as well as robust antibacterial activity, especially against multidrug-resistant bacteria in vitro and in vivo. The nanocomposites hydrogel also demonstrates outstanding photothermal performance with (near-infrared laser irradiation) NIR irradiation, which could effectively kill the tumor cell $(>90 \%)$ and inhibit tumor growth (inhibition rate up to $94 \%$ ) in a subcutaneous skin-tumor model. In addition, the nanocomposites hydrogel effectively accelerates wound healing in vivo. These results suggest that the BGN-based nanocomposite hydrogel is a promising candidate for skin-tumor therapy, wound healing, and antiinfection. This work may offer a facile strategy to prepare multifunctional bioactive hydrogels for simultaneous tumor therapy, tissue regeneration, and anti-infection.

\section{Introduction}

Skin plays an important role in keeping body homeostasis and protecting body from many noxious substance. ${ }^{[1]}$ Skin cancer is diagnosed over a million cases every year, which showed high incidence in western countries. ${ }^{[2,3]}$ The current treatment of skin tumor in clinic mainly includes surgical excision, chemo/radiotherapy and immunotherapy. ${ }^{[3-5]}$ However, many normal skin tissues around the skin-tumor cells must be cleared to prevent recurrence, which results in large skin defects followed by infection and chronic wound healing. ${ }^{[4]}$ Additionally, the traditional chemo/radiotherapy has been widely used to avoid recurrence, but the severe side effect and resistance cause the endless pain to patients..$^{[5,6]}$ Until now, it is a major challenge to fulfill the cancer therapy, wound healing and anti-infection after surgical remove of tumor. ${ }^{[7]}$ Specially, multidrug resistant bacterial infection was one of the key obstacles in chronic wound healing. ${ }^{[8]}$ Therefore, it is significant for

Dr. L. Zhou, Y. Liu, Prof. B. Lei

Key Laboratory of Shaanxi Province for Craniofacial

Precision Medicine Research

College of Stomatology

Xi'an Jiaotong University

Xi'an 710049, China

E-mail: rayboo@xjtu.edu.cn

Dr. L. Zhou, Y. Xi, Y. Xue, M. Wang, Y. Guo, Prof. B. Lei

Frontier Institute of Science and Technology

Xi'an Jiaotong University

Xi'an 710049, China

Y. Guo

Department of Biomedical Engineering

University of Michigan

Ann Arbor, MI 48109, USA

Prof. B. Lei

Instrument Analysis Center

Xi'an Jiaotong University

Xi'an 710049, China

The ORCID identification number(s) for the author(s) of this article can be found under https://doi.org/10.1002/adfm.201806883.

DOI: $10.1002 / \mathrm{adfm} .201806883$ designing novel biomaterials for treating cancer, accelerating wound healing, and multidrug resistant bacterial infection. To the best of our knowledge, there are very few biomaterials with such three functions for efficient skin-tumor therapy.

In recent years, photothermal therapy (PTT) has been emerged as a promising strategy to treat various cancers due to the high efficiency and low toxicity. ${ }^{[9,10]}$ Various photothermal agents have been extensively developed, including many inorganic nanomaterials (gold nanostructures and carbon-based nanomaterials) and organic PTT agents such as peptide- and protein-based nanomaterials, micelles containing NIR (nearinfrared) dye, organic/inorganic nanocomposites and conjugated polymers, and so on. ${ }^{[11-16]}$ Polydopamine (PDA) has been employed as important biomaterials based on its excellent biocompatibility and lower toxicity in vitro and in vivo. Specially, PDA could be used for the tumor therapy owing to its good photothermal performance. ${ }^{[17-19]}$ Due to the mussel-inspired characteristic, PDA was easily to be deposited on the surface of nanoparticles. PDA functionalized nanoparticles are also easily to conjugate with other biopolymers through the amidation or 
Schiff base reaction with PDA. Then, the uniform nanocomposites with multifunctional properties could be fabricated via the PDA-based chemistry. ${ }^{[18-21]}$ The multifunctional hybrid hydrogel based on PDA was also reported for various biomedical applications. ${ }^{[22-24]}$

Silicon-based bioactive biomaterials such as bioactive glass (BG, typical composition: $\mathrm{SiO}_{2}-\mathrm{CaO}-\mathrm{P}_{2} \mathrm{O}_{5}$ ), have been extensively used for bone tissue repair, owing to its good biocompatibility, biodegradation, tissue regeneration ability. ${ }^{[25-34]}$ Monodispersed BG nanoparticles (BGNs) with enhanced biological performance were also developed for bioimaging, osteogenic differentiation of stem cells, drugs, and gene delivery. ${ }^{[32-38]}$ Recently, it was shown that nanoscale BG could enhance chronic wound healing through improving the angiogenesis. ${ }^{[38,39]}$ Thus, various biodegradable nanocomposites based on BGNs were fabricated to meet the increased requirement of tissue repair and regeneration. ${ }^{[40,41]}$

Hydrogels based on biodegradable biomaterials have become a promising tool for biomedical application owing to their biomimetic mechanical environment and easily preparation to fit special needs. ${ }^{[42-47]}$ Some multifunctional hydrogels and novel biocompatible polymers have been developed to drug delivery, cancer therapy, and bacterial infection and wound healing. ${ }^{48-53]}$ However, multifunctional hydrogel with efficient anti-infection ability especially multidrug-resistant antibacterial activity, skintumor therapy, and wound healing are few reported. ${ }^{[44]}$

Herein, we develop a multifunctional bioactive nanocomposites hydrogel with self-healing and antibacterial capacity against multidrug resistant bacteria for PTT of skin tumor and wound healing. The multifunctional hydrogel was fabricated through the click chemical crosslinking of F127- $\varepsilon$-Poly-L-lysine (FEPL), F127-Phe-CHO and BGN@PDA (FCB hydrogel, F: FEPL; C: F127-Phe-CHO; B: BGN@PDA). In this strategy, BGN@PDA was designed to achieve photothermal effect and stimulate the skin repair. FEPL was used to anti-infection, originating from EPL which showed high antibacterial activity in our previous report, ${ }^{[54]}$ and could form the hydrogel network with F127-PheCHO and BGN@PDA through the Schiff base reaction. The synthesis, physicochemical structure and properties, biomedical functions of FCB hydrogel in skin cancer treating, antiinfection, and skin repair were totally investigated.

\section{Results and Discussion}

\subsection{Fabrication and Characterization of FCB Hydrogel}

The synthesis process and application in tumor therapy and wound healing of FCB hydrogel are shown in Figure 1. As shown in Figure 1A, F127-Phe-CHO and FEPL were synthesized by the reaction of F127-OTs, 4-hydroxybenzaldehyde and EPL, respectively. BGN was decorated with PDA by oxidation and polymerization of DA on its surface ${ }^{[55]}$ (Figure 1B). FCB hydrogel was performed through a Schiff base process between the amino group of FEPL, the aldehyde group of F127-Phe-CHO and BGN@PDA, respectively. Additionally, it was anticipated that FCB hydrogel has excellent antibacterial ability, effective NIR-induced photothermal tumor therapy, and enhanced wound healing ability in vivo (Figure 1C).
The chemical structures of the obtained F127-OTs, F127-Phe$\mathrm{CHO}$, and FEPL were elucidated by ${ }^{1} \mathrm{H}$ nuclear magnetic resonance (NMR) and Fourier-transform infrared (FTIR) (Figure S1, Supporting Information and Figure 2). In ${ }^{1} \mathrm{H}$ NMR spectra, the peaks at $\delta 7.91,7.29$ and $2.48 \mathrm{ppm}$ were belonged to the aromatic ring and methyl from $\mathrm{TsCl}$ and other strong peak at $\delta 1.12 \mathrm{ppm}$ was attributed to the methyl of F127, indicating the formation of F127-OTs (Figure S1A, Supporting Information). The absence of methyl peak (2.48 ppm), new resonance signals of the aromatic ring at $\delta 7.81$ and $7.01 \mathrm{ppm}$, aldehyde group at $\delta 9.87 \mathrm{ppm}$, suggested the formation of F127-Phe-CHO (Figure S1B, Supporting Information). In Figure 2A, the disappearance of proton peaks of aromatic ring and five new proton signals at $\delta 3.79$, $3.15,1.79,1.50$, and $1.31 \mathrm{ppm}$ assigned as the methine and methylene groups of EPL, confirmed the formation of FEPL copolymer. The graft ratio of $-\mathrm{CHO}(\approx 100 \%)$ and EPL $(\approx 100 \%)$ in F127 were calculated according to the integral values of peaks at $\delta 1.12 \mathrm{ppm}$ (the methyl of F127), $\delta 9.87 \mathrm{ppm}$ (-CHO), and $\delta$ $3.15 \mathrm{ppm}$ (methylene groups of EPL), respectively.

The chemical structures of F127-OTs, F127-Phe-CHO, FEPL, FCE, and FCB hydrogel were further confirmed by FTIR (Figure 2B). The skeletal vibration of aromatic ring of F127-OTs was at $1600 \mathrm{~cm}^{-1}$. The synthesis of F127-OTs could be confirmed thorough the peaks at 2876 and $1099 \mathrm{~cm}^{-1}$ assigned the $\mathrm{C}-\mathrm{H} / \mathrm{ether}$ stretch bands from F127. The symmetric vibration of carbonyl in aldehyde group of F127-Phe-CHO appeared at $1687 \mathrm{~cm}^{-1}$. According to the FTIR of the EPL, the absorption of ether bands $\left(1099 \mathrm{~cm}^{-1}\right)$ was expected for F127-OTs. The disappearance of skeletal vibration of aromatic and presence of -NH- stretching band of EPL $\left(3247 \mathrm{~cm}^{-1}\right)$ suggested the formation of FEPL. In the FTIR spectrum of the FCE hydrogels, the disappearance of bands at $1687 \mathrm{~cm}^{-1}$ (aldehyde) and the new peak at $1662 \mathrm{~cm}^{-1}(\mathrm{C}=\mathrm{N})$, suggesting the Schiff base reaction between $-\mathrm{CHO}$ groups of F127-Phe-CHO and $-\mathrm{NH}_{2}$ of FEPL. ${ }^{[62]}$

However, in FCB hydrogel, due to the probable similar crosslinking mechanism between BGN@PDA- F127-Phe-CHO and FEPL-F127-Phe-CHO, therefore, no significant change was observed in the FTIR spectrum of FCE and FCB hydrogels. To demonstrate the interaction between BGN@PDA and F127-Phe-CHO, the X-ray photoelectron spectroscopy (XPS) analysis was conducted. Additionally, the results of XPS showed that the ratio of oxygen to nitrogen in FCB hydrogel (ca. 2.94, Figure S2A, Supporting Information) was lower than that of in FCE hydrogel (ca. 3.48, Figure S2B, Supporting Information), confirming the interaction between F127-EPL and BGN@PDA in FCB hydrogel, which leaded to the lower ratio owing to the consumption of oxygen in the reaction. There was no significant difference in crystalline composition between FCB and FCE (Figure S2C, Supporting Information).

The monodispersed PDA-coated BGNs (BGN@PDA) were synthesized (shown in SI) and confirmed by UV-vis-NIR and transmission electron microscope (TEM) analysis. UV-vis spectra displayed an absorption peak at $\approx 415 \mathrm{~nm}$, belonging to PDA (Figure S1C, Supporting Information). The BGN@ PDA showed significant NIR absorption between 800-1000 nm, predicted its potential photothermal property (Figure S1C, Supporting Information). BGN displayed monodispersed mesoporous nanostructure $(\approx 300 \mathrm{~nm}$ in diameter) (Figure S1D, 

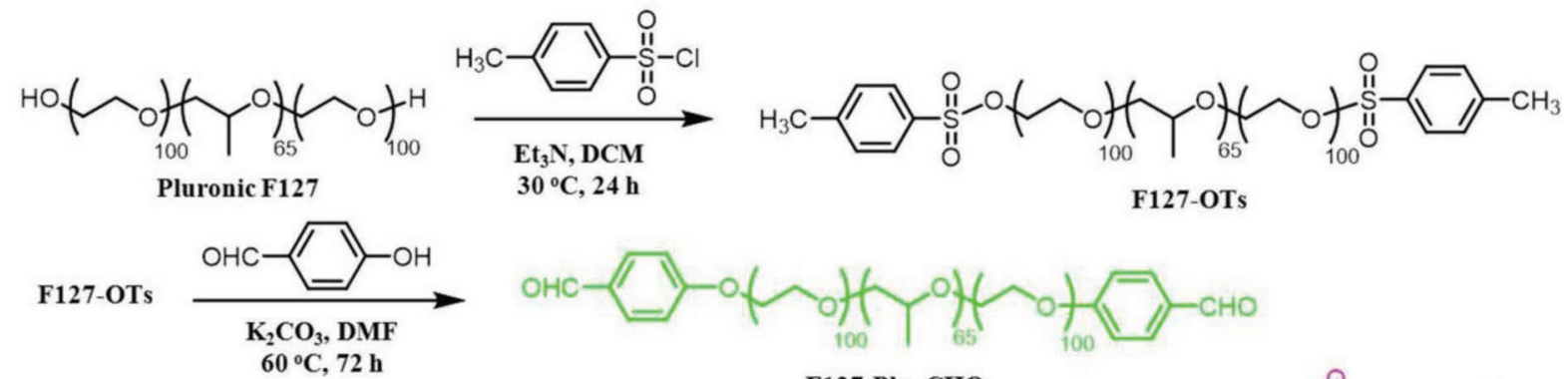

F127-Phe-CHO<smiles>NCCCCC(N)C(=O)O</smiles>

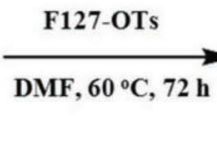<smiles>CNCCCCC(NOC(C)COC(C)C)C(=O)O</smiles><smiles>CCOC(C)(C)COC(O)NC(CCCCN)C(=O)O</smiles>

FEPL
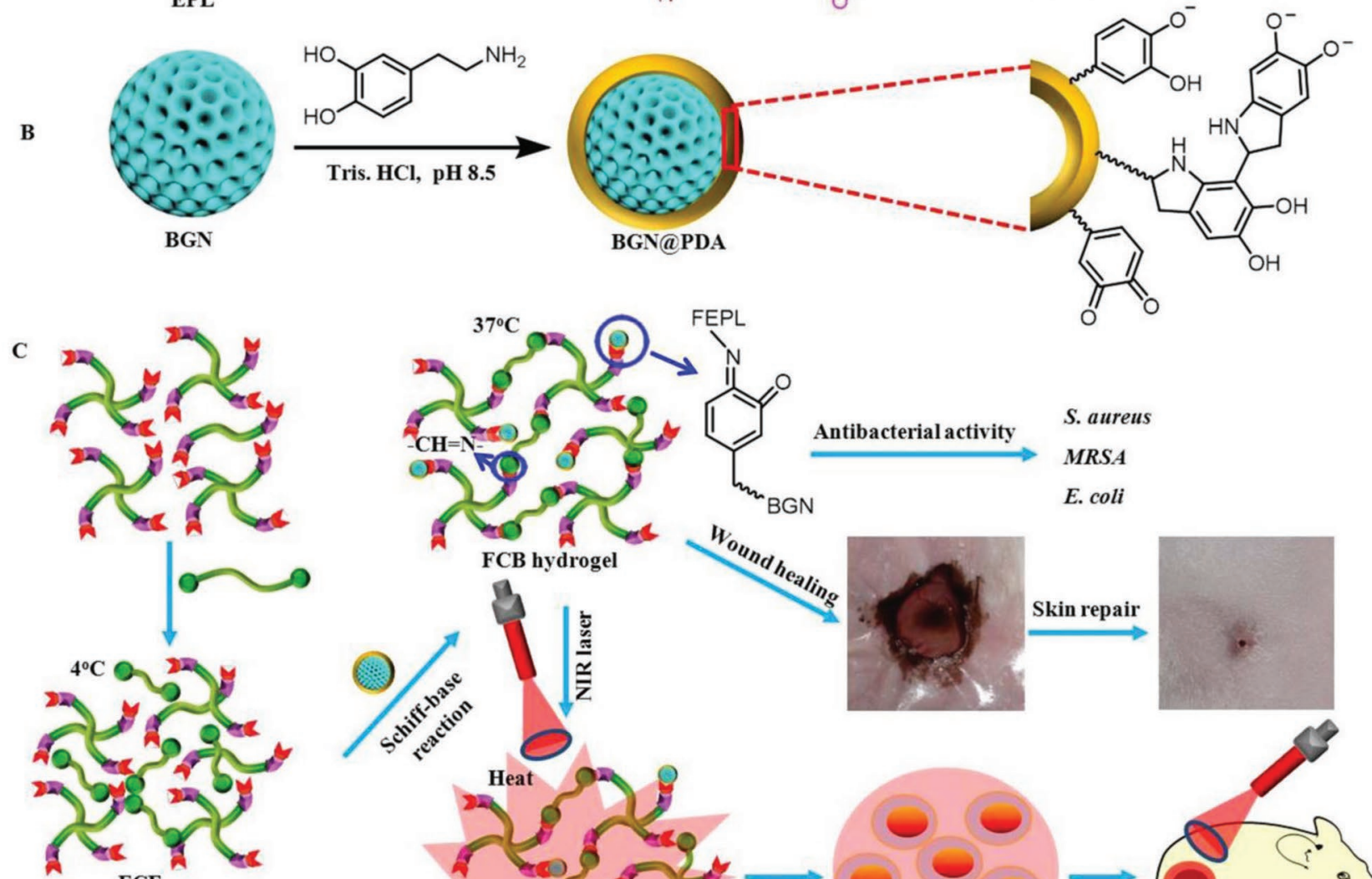

FCE
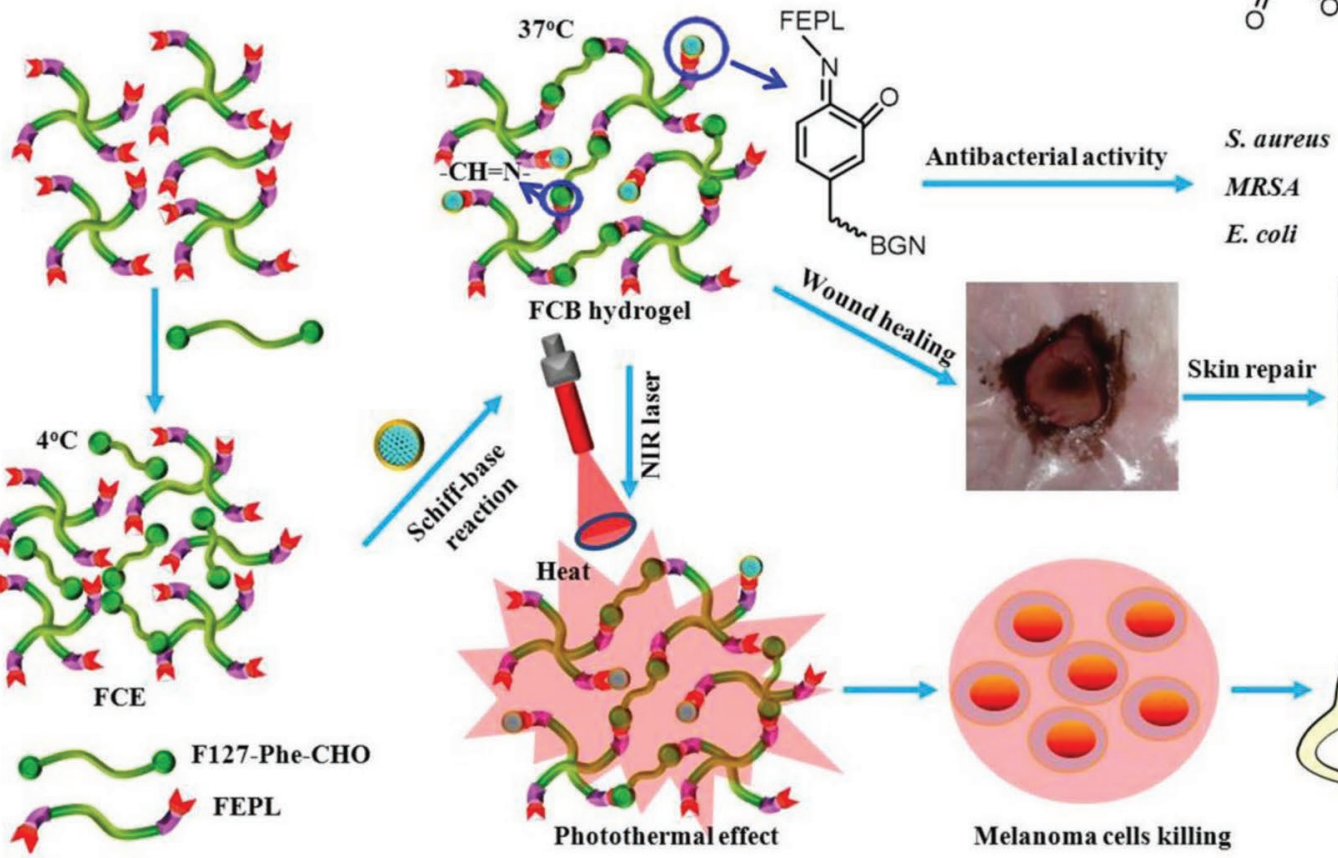

Figure 1. Scheme showing the preparation and application of FCB hydrogel in tumor therapy and wound healing. A) The synthesis route of F127-OTs and FEPL. B) Schematic illustration for the formation of FCB hydrogel and bioapplication.

Supporting Information). Additionally, BGN@PDA showed that a continuous and smooth membrane with a thickness of $\approx 20 \mathrm{~nm}$ was coated on the surface of the BGN, indicating the formation of a layer of PDA film (Figure S1D, Supporting Information). After freeze-drying, FCE and FCB hydrogels showed representative porous structures. By comparison with the image of FCE, some BGN@PDA particles could be found in the image of FCB (Figure 2C). Additionally, the characteristic peaks of Si in the EDS spectrum of FCB hydrogel further confirmed the existence of BGN@PDA in the obtained hydrogels (Figure 2D).

\subsection{Rheological, Self-Healing and Injectable Properties}

The sol-gel transformation temperatures in hydrogels were important for the biomedical applications including tumor 
A

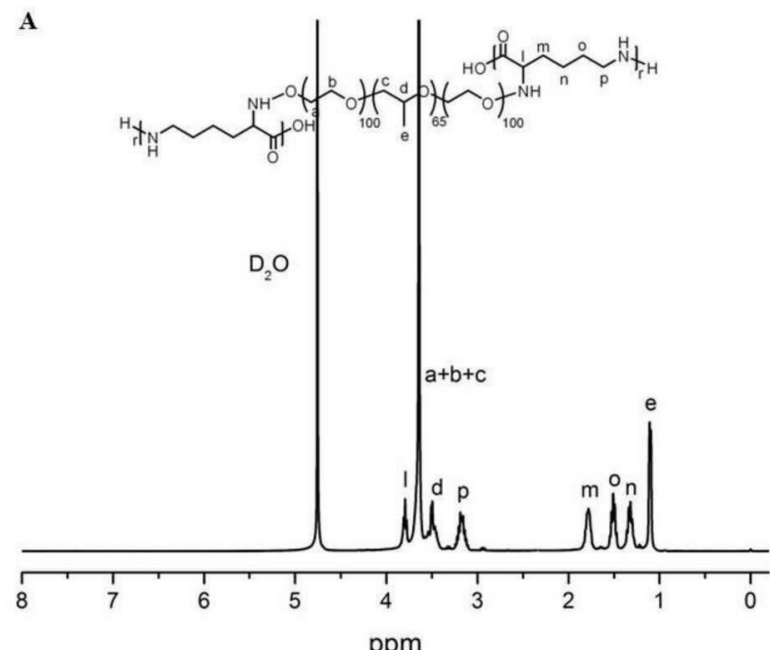

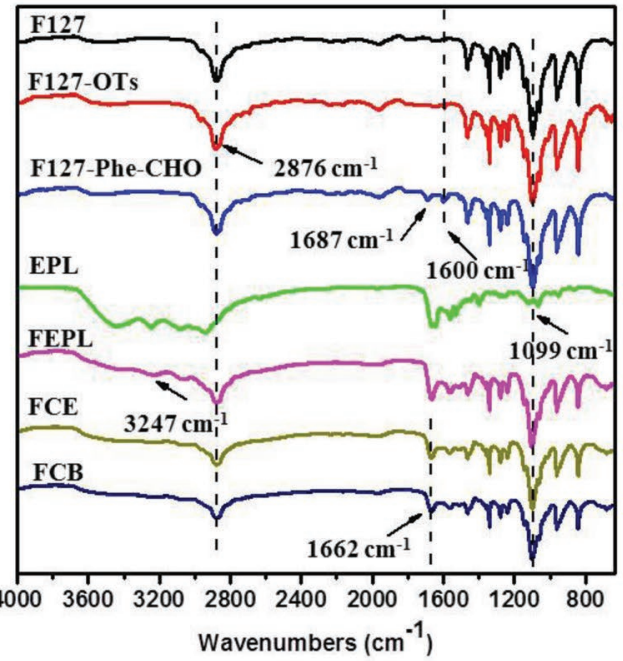
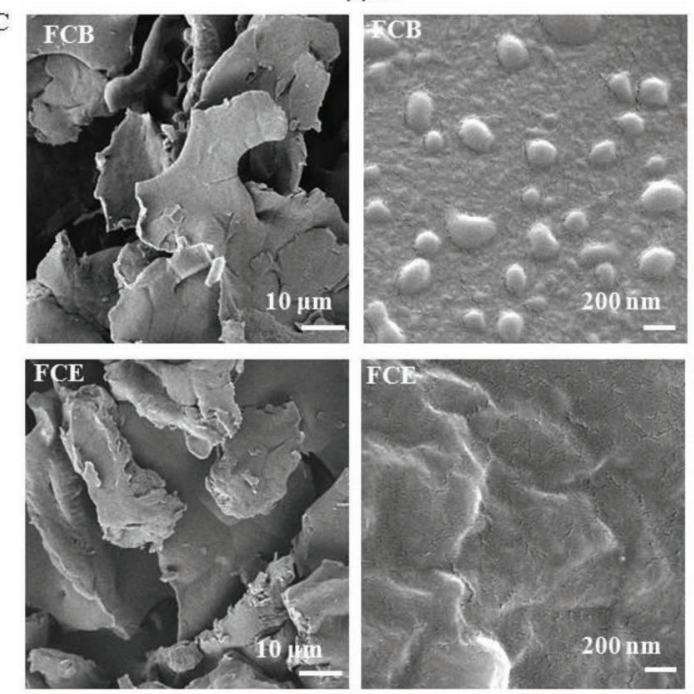

D
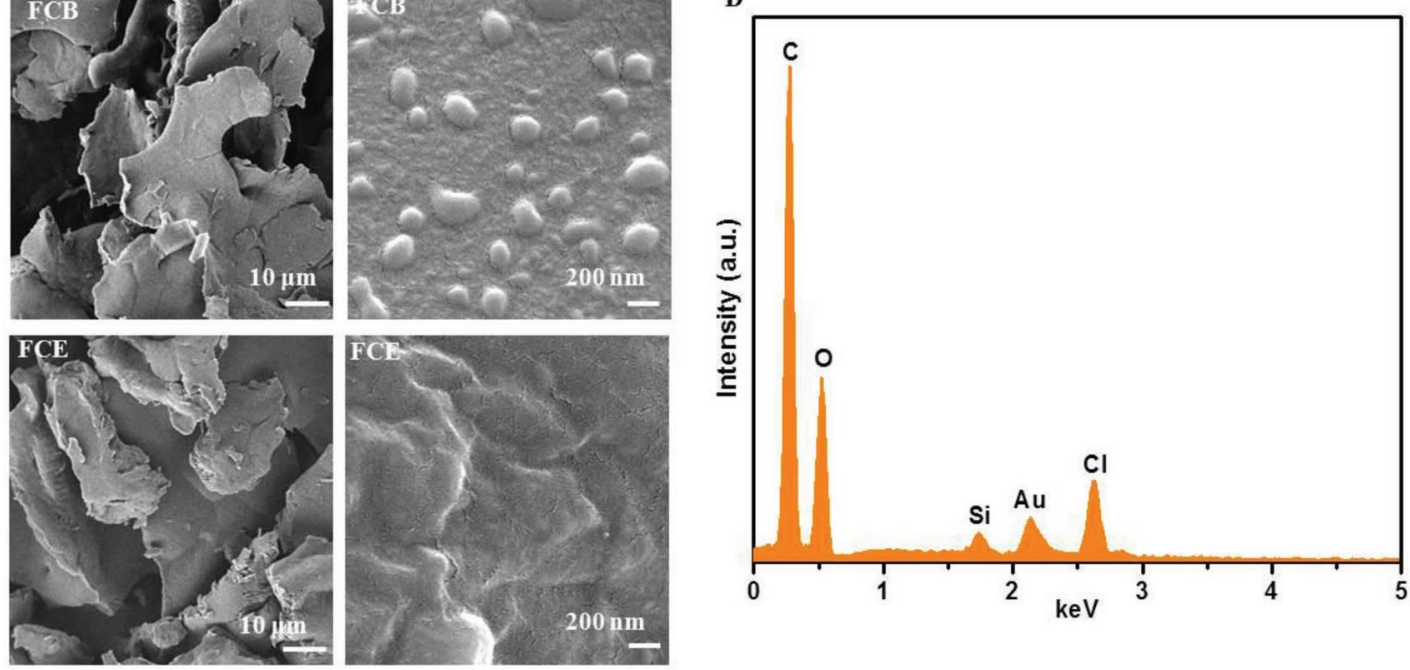

Figure 2. Characterization. A) ${ }^{7} \mathrm{H}$ NMR spectra of FEPL in $\mathrm{D}_{2} \mathrm{O}$; B) FTIR of precursors and FCB hydrogel; C) Field emission scanning electron microscopy (FESEM) images of FCE and FCB hydrogels; and D) EDS spectrum of FCB hydrogel.

therapy and wound healing. ${ }^{[56,57]}$ To evaluate the gelation temperature of the FCE and FCB hydrogels, dynamic temperature sweep rheological experiments were conducted to monitor the gelation process. The storage modulus $\left(G^{\prime}\right)$ values in FCB and FCE were greater than the loss moduli $\left(G^{\prime \prime}\right)$ when the temperature was more than $30^{\circ} \mathrm{C}$, indicating the formation of hydrogels (Figure S3, Supporting Information). The crossover of modulus ( $G^{\prime}$ and $\left.G^{\prime \prime}\right)$ of FCB was about $27.8^{\circ} \mathrm{C}$, which was lower than that of FCE (about $30.5^{\circ} \mathrm{C}$ ), suggesting that the BGN@PDA could contribute to the formation of hydrogels by the reaction of PDA and $-\mathrm{NH}_{2}$ of FEPL as described in Figure 1 . At $25^{\circ} \mathrm{C}$, the $G^{\prime \prime}$ of FCB was higher than that of $G^{\prime}$, indicating its solution state (Figure $3 \mathrm{~A}$ ). When the temperature reached $37^{\circ} \mathrm{C}$, FCB became to gel state (approximately $55 \mathrm{~s}$ ) (Figure 3C) owing to the fact of $G^{\prime}>G^{\prime \prime}$, suggested the temperature-responsive property of FCB hydrogel (Figure 3A,C). Moreover, the $G^{\prime}$ and $G^{\prime \prime}$ of FCB were higher than that of FCE at $37^{\circ} \mathrm{C}$, further confirming the synergies of BGN@PDA in the formation of FCB hydrogel networks (Figure S3, Supporting Information). The shear-thinning behavior of FCB hydrogel was confirmed by the rheological analysis under high and low shear strain (Figure 3B). The network of the FCB hydrogels were disrupted by the high shear strain $(1000 \%)$, resulting in a significant decrease in $G^{\prime}$ to a level below $G^{\prime \prime}$. At the low shear strain (1\%), the $G^{\prime}$ for FCB hydrogels were completely recovered immediately (Figure 3B), even after three cycles, demonstrating their rapid self-healing and shear-thinning capacity. The self-healing ability of FCB hydrogel was also showed through morphological repair experiment in which a cavity created in hydrogel. After $6 \mathrm{~h}$, the cavity of hydrogel became smaller and disappeared completely after $12 \mathrm{~h}$, indicating the good self-healing capability of FCB hydrogel (Figure 3D). In addition, the shear-thinning behavior and the excellent self-healing of FCB hydrogel enabled the facile injection capacity without clogging and writing smoothly (Figure 3E).

\subsection{Photothermal Properties and Cytotoxicity Evaluation of FCB Hydrogel}

The temperature changes $\left(\Delta_{t}\right)$ of FCB hydrogel was promoted dramatically up to about $40{ }^{\circ} \mathrm{C}$ during 9 min (Figure 4A,B). 
A

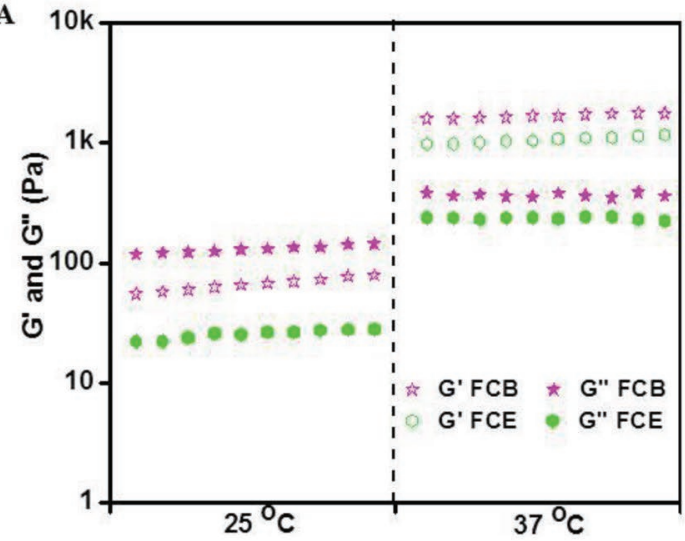

B

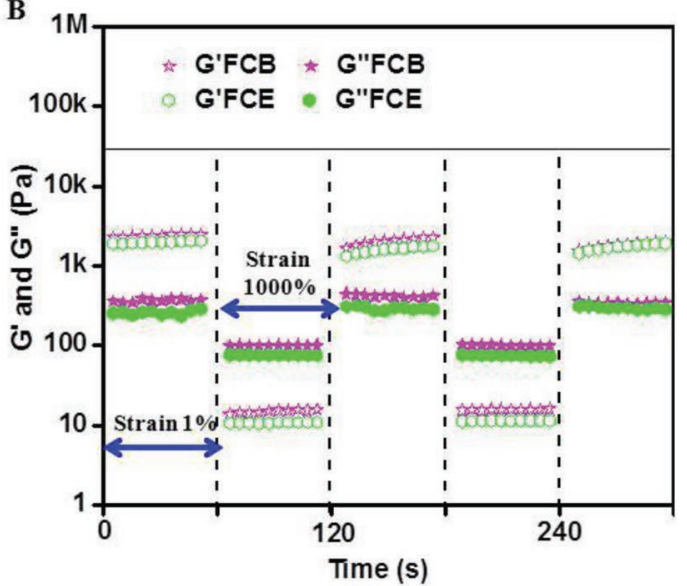

C
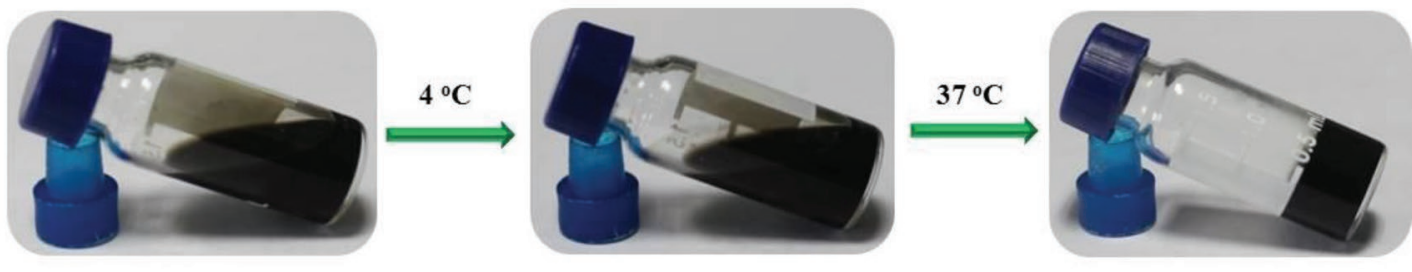

D

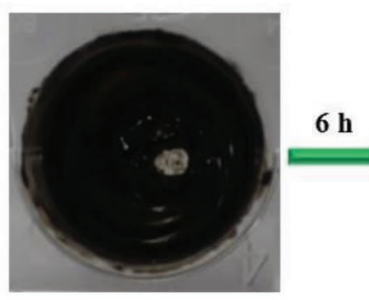

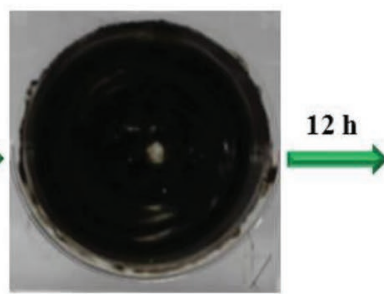

Self-healing process
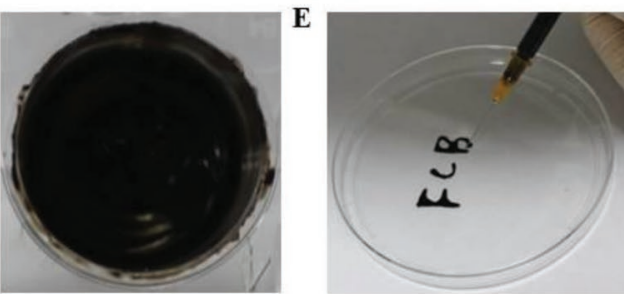

Injectability

Figure 3. A) Rheological analysis of the $\mathrm{FCB}$ hydrogels at $25^{\circ} \mathrm{C}$ and $37^{\circ} \mathrm{C}$; B) rheological data of $\mathrm{FCB}$ hydrogel under alternating high (1000\%) and low shear (1\%); C) the images of the FCB hydrogel forming; and D,E) images of self-healing and injectability of the FCB hydrogels.

By contrast, the FCE hydrogel and $\mathrm{H}_{2} \mathrm{O}$ indicated poor temperature changes at the same conditions. FCB hydrogel without NIR irradiation showed no changes in temperature and thermal image. These results displayed that the excellent photothermal performance of FCB hydrogel was due to the incorporation of BGN@PDA. The anticancer efficiency of FCB hydrogel in vitro was evaluated in A375 cancer cells. The live/dead images of cells showed that a large number of dead cells (red fluorescence) were observed in FCB + NIR group (Figure 4C). By comparison, the cells in the other five groups were almost alive (green fluorescence, Figure 4C). The cell viability in FCB + NIR group (10 $\mathrm{min})$ was decreased to $18 \%$, significantly lower than that of controls (NC, FCB, and NC+ NIR group, Figure 4D). The cell viability of FCB + NIR was further decreased with the increasing of irradiation duration (9.3\% for $20 \mathrm{~min}$ ). Additionally, the cell viability of hydrogels (FCB and FCE) in A375 and $\mathrm{C} 2 \mathrm{C} 12$ cells were greater than $80 \%$ even incubation for 24 or $48 \mathrm{~h}$, showing their lower cytotoxicity. These results demonstrated the excellent photothermal therapeutic efficiency of FCB hydrogel with better biocompatibility in vitro.

\subsection{Antibacterial Capacity Evaluation In Vitro/In Vivo}

The bacterial infection after surgical tumor therapy has been the one of major obstacles in wounding healing, ${ }^{[58,59]}$ therefore, the antibacterial efficacy of biomaterials is very important. FCB hydrogel exhibited considerable antibacterial-killing ability for $E$. coli and $S$. aureus, which was equivalent to that of FCE (99\%), suggesting that BGN@PDA did not affect the antibacterial efficacy of FCB (Figure 5A,B). In contrast, BGN@PDA and ampicillin showed weak antibacterial activity, in which the highest killing efficiencies were $63 \%$ and $56 \%$ against $S$. aureus, respectively (Figure $5 B$ ). In addition, FCB hydrogel also showed higher antibacterial activity against MRSA, in which the killing efficiency was up to $99 \%$, whereas the corresponding value of ampicillin was 24\% (Figure 5B).

The anti-infection efficacy of FCB hydrogel in vivo was also assessed using MRSA-infected mouse as the model (Figure 5C-E). The mice were infected by MRSA through subcutaneous injection and were randomly divided into five groups $(n=5)$ after $3 \mathrm{~d}$ infection. The antibacterial efficacy was evaluated by counting the number of bacteria came from the 

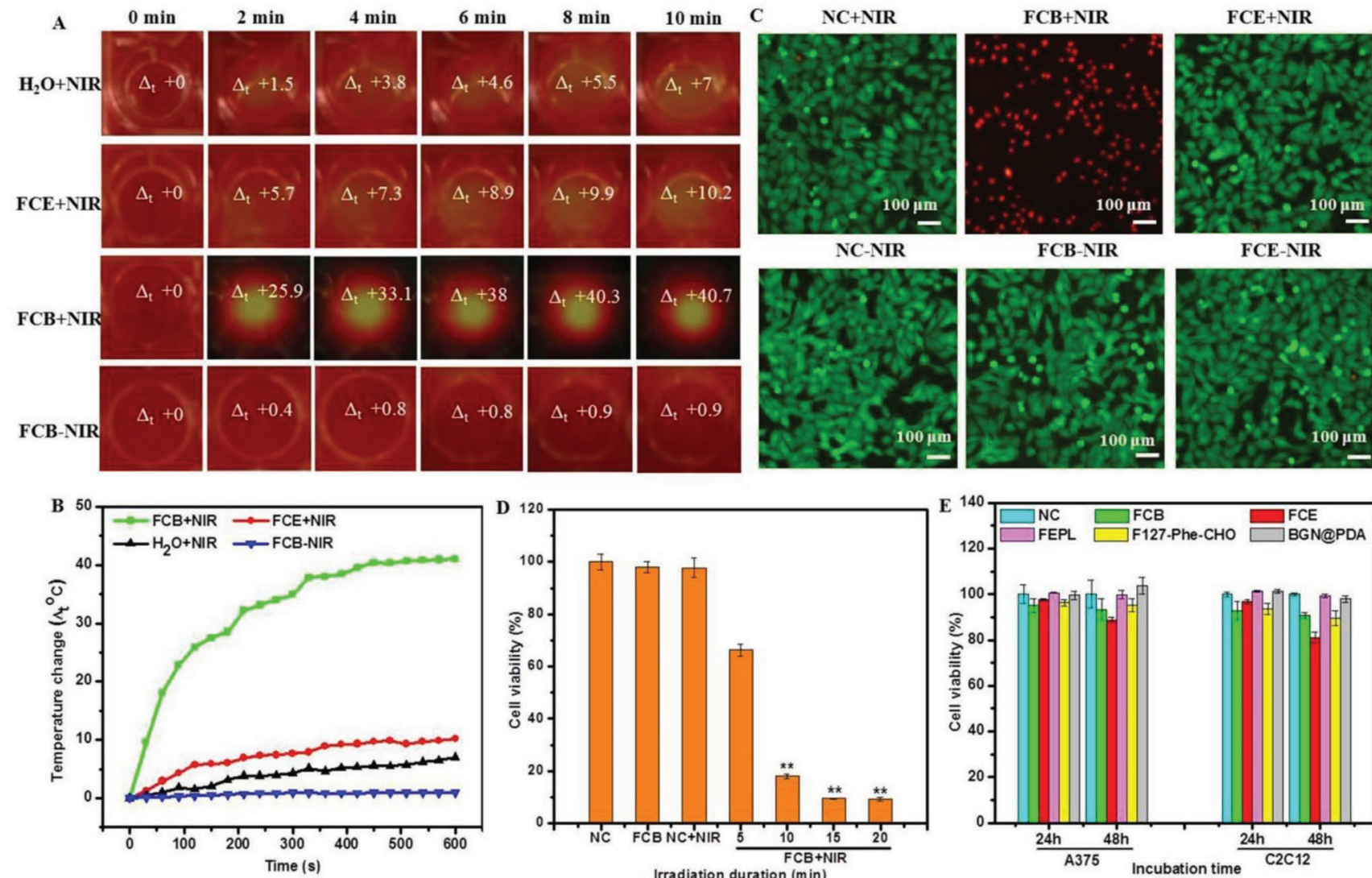

FCE-NIR

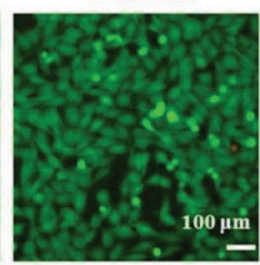

Figure 4. Photothermal performance and in vitro anticancer efficiency. A,B) The real-time infrared thermal images and photothermal heating curves of FCB hydrogel and controls with NIR irradiation $\left.\left(808 \mathrm{~nm}, 1.41 \mathrm{~W} \cdot \mathrm{cm}^{-2}\right) ; C\right)$ in vitro anticancer efficiency of FCB hydrogel and controls with or without NIR treatment $\left.\left(808 \mathrm{~nm}, 1.41 \mathrm{~W} \cdot \mathrm{cm}^{-2}, 10 \mathrm{~min}\right) ; \mathrm{D}\right) \mathrm{FCB}$ hydrogel under different irradiation duration $\left(808 \mathrm{~nm}, 1.41 \mathrm{~W} \cdot \mathrm{cm}^{-2}\right)(* p<0.05, * * k<0.01$,

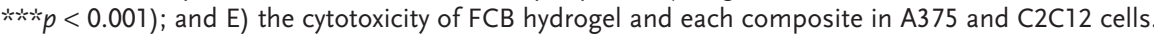

tissue homogenates plated onto LB-agar plates (Figure 5C, D). Mice treated with ampicillin twice displayed a bacterial with $2.1 \times 10^{7} \mathrm{CFU} \mathrm{mL} \mathrm{m}^{-1}$, similar with control (phosphate buffered saline (PBS)-treated group, Figure 5D). By comparison, FCBtreated group revealed significant less bacteria, in which the

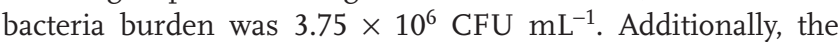
antibacterial efficacy of FCB $(\approx 84 \%)$ was significantly higher than that of FCE ( $\approx 78 \%)$ and BGN@PDA ( $\approx 51 \%)$, suggesting that FCB displayed higher antibacterial activity with synergistic effect of FCE and BGN@PDA (Figure 5C,D). As depicted in Figure $5 \mathrm{E}$, the inflammatory exudates and the macrophages in the tissue treated by FCB hydrogel was much less than others, demonstrating that the MRSA infection in vivo was significantly reduced. The antibacterial performance of FCB hydrogel was probably originated from EPL which showed high antimicrobial ability against various bacterial. ${ }^{[60,63]}$

\subsection{In Vivo Antitumor Effect of FCB Hydrogel}

The antitumor efficacy of FCB hydrogel was further confirmed in nude mice with A375 tumor xenografts. After irradiation for $10 \mathrm{~min}$ on tumor with various groups, the temperature change $\left(\Delta_{t}\right)$ was approximately $5{ }^{\circ} \mathrm{C}$ for PBS + NIR group and
FCE + NIR groups, and FCB+NIR group showed the rapid improvement for $\Delta_{t}\left(16{ }^{\circ} \mathrm{C}\right)$ (the temperature of tumor was about $51{ }^{\circ} \mathrm{C}$, Figure $\left.6 \mathrm{~A}, \mathrm{~B}\right)$. The tumor volume of FCB + NIR group was reduced significantly after PTT for $4 \mathrm{~d}$, while the control groups presented a rapid tumor growth (Figure 6C,D). The tumor volume in FCB + NIR group was far lower than other three controls, which was reduced by $94 \%$ compared to PBS on day 18 (Figure 6C,D). Additionally, the tumors of FCB + NIR group were gradually disappeared without recurrence after treatments (day 18, Figure S4, Supporting Information). By comparison, FCB without NIR irradiation had no effect on tumor inhibition, which showed the comparable tumor volume with PBS + NIR and FCE + NIR groups (Figure 6C,D). Moreover, after $18 \mathrm{~d}$ treatment, the mean tumor weight in FCB + NIR group was the smallest among all groups, indicating that FCB hydrogel with NIR efficiently suppressed tumor growth (Figure 6E).

H\&E and terminal deoxynucleotidyl transferase dUTP nick end labeling (TUNEL) staining were used to evaluate the microscopic therapeutic efficacy of FCB hydrogel in vivo. A lot of apoptotic tumor cells were seen in FCB + NIR group, while cells in controls were well alive (Figure 6F). In addition, $\mathrm{TUNEL}^{+}$apoptotic tumor cells of the FCB + NIR group was much more than other groups as shown in quantitative 

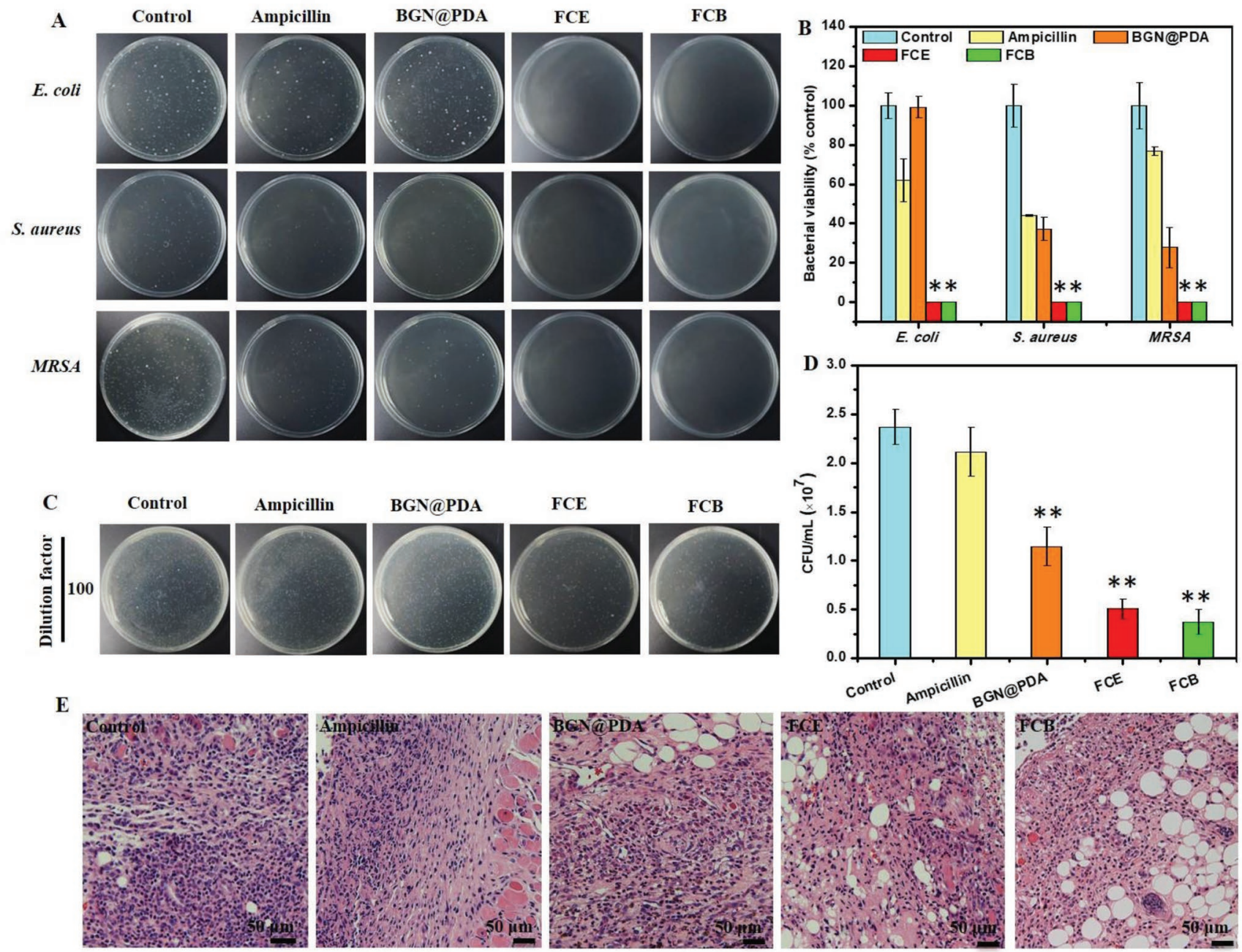

Figure 5. Antibacterial activity in vitro and in vivo. A) Pictures of agar plates and B) corresponding statistical data of colonies densities of E. coli, S. aureus, and MRSA treated with different samples ( $* p<0.05$ and $* * p<0.01$ ). C) Images of MRSA colonies growing on the agar plates come from the homogenized infected tissues after PBS buffer (Control), Ampicillin, BGN@PDA, and FCE and FCB treatments; D) quantitative bacterial colonies densities based on C) ( $* p<0.05$ and $* * p<0.01$ ); E) optical images of H\&E stained tissue sections after treated with PBS buffer (Control), Ampicillin, BGN@PDA, FCE, and FCB, respectively (scale bars: $50 \mu \mathrm{m}$ ).

analysis (Figure 6G). These results indicated that FCB hydrogel could significantly inhibit tumor development by promoting apoptosis of cancer cells in vivo owing to its NIR-induced hyperthermia.

\subsection{In Vivo Wound Healing of FCB Hydrogel}

The potential wound healing efficacy of FCB hydrogel was further performed in mice with full-thickness skin wound, using FCE and commercial dressing (3M) as controls. FCB hydrogels presented a higher wound healing rate relative to other groups $\left({ }^{*} p<0.05\right.$, Figure 7). As compared to FCE and 3M group, on day 3 after surgery, the wound area of FCB group was slightly reduced and the elevated re-epithelialization was obviously detected (Figure 7A,B). The relative wound areas on day 7 for the FCB hydrogel, FCE, 3M, and Blank was 33.1\%, 53.5\%, $77.6 \%$, and $52.5 \%$, respectively (Figure $7 \mathrm{~B}$ ). The wound defects filled with FCB were almost healed on day 10, in which the relative wound area was $8.3 \%$, by comparison, $29.2 \%, 40.7 \%$, and $31.8 \%$ for FCE, $3 \mathrm{M}$, and Blank groups, respectively. The remaining wound area on day 14 for FCB (1.2\%) was much lower than FCE (4.3\%), 3M (24.5\%), and Blank groups (13.4\%), demonstrating that the wound was almost completely healed (Figure 7A,B).

Additionally, the H\&E images showed that the epidermis of FCB group was gradually appeared with the increasing of time (Figure S5, Supporting Information). On day 14, relative to other groups, abundant granulation tissue (black arrows) was observed in FCB group (Figure 7C). Granulation tissue included extracellular matrix, fibroblasts, and many growth factors, which was beneficial for wound healing process. The granulation tissue thickness in FCB hydrogel group displayed approximately $240 \mu \mathrm{m}$ thicker than controls (Figure S5B, Supporting Information). Masson's trichrome staining indicated that more positive staining could be observed in FCB 

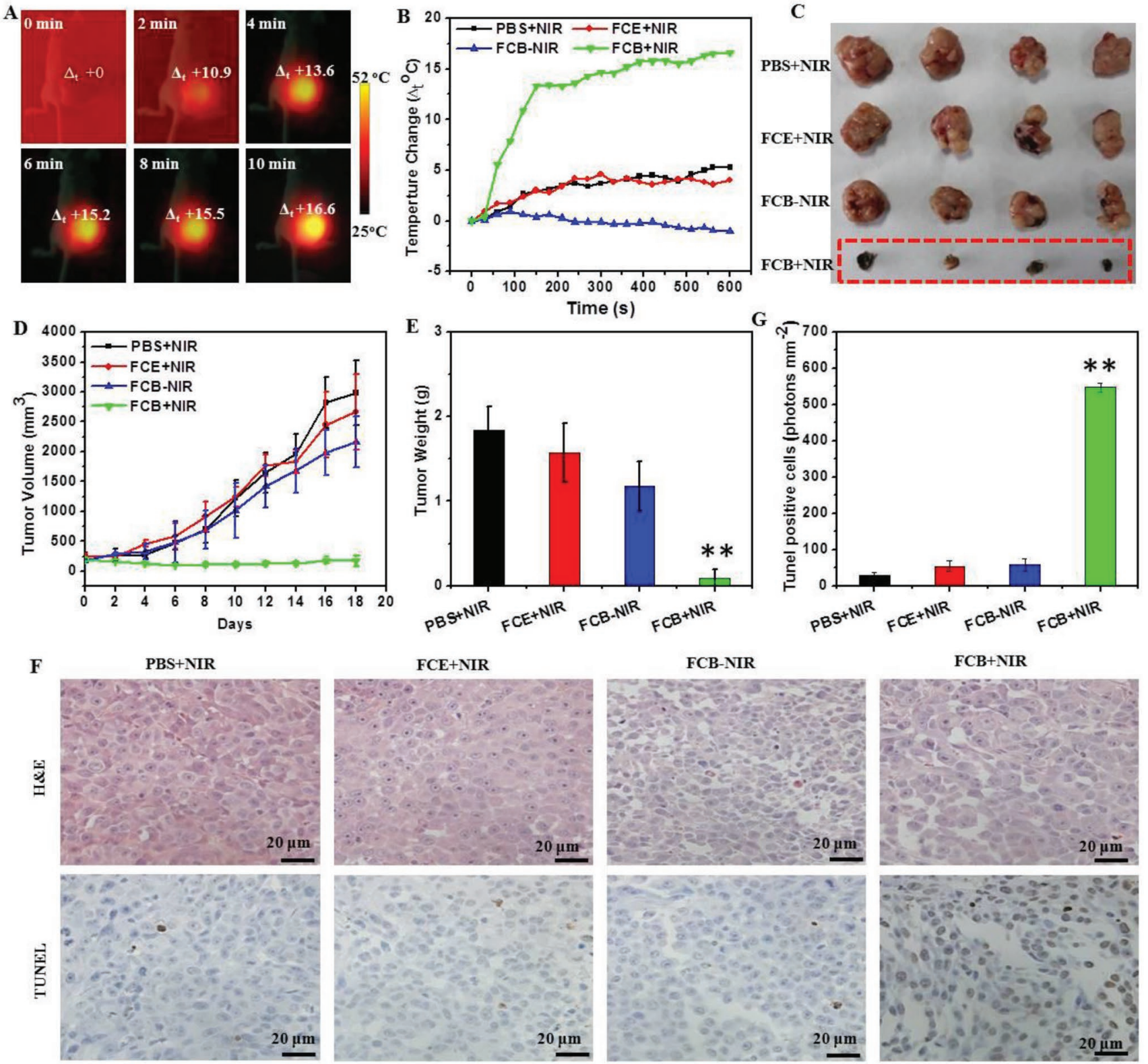

Figure 6. In vivo PTT of FCB hydrogel. A) NIR thermal images of FCB hydrogel and B) photothermal heating curves of A375 tumor-bearing mice treated with FCB, FCE, and PBS when treated with or without irradiation $\left(808 \mathrm{~nm}, 1.41 \mathrm{~W} \cdot \mathrm{cm}^{-2}, 10 \mathrm{~min}\right)$; C) photographs of the tumors after various treatments on day 18; D) tumor volume and E) weight at the end of experiment; F) images of H\&E and TUNEL analyses of tumor tissues after treatment for $18 \mathrm{~d}$ with various formulations. ( $* p<0.05$ and $* * p<0.01$, Scale bar: $20 \mu \mathrm{m}$ ); and G) Tunel ${ }^{+}$tumor cells in tumor sections quantified by Image-).

group (Figure S6B, Supporting Information), suggesting more collagen content in FCB group (Figure 7C and Figure S6, Supporting Information). Additionally, the immunohistochemical staining of vascular endothelial growth factor (VEGF) expression was also performed to analyze the angiogenesis in wound healing. There were more VEGF positive vessels (brown, black arrows showed) observed in the FCB group than the controls (Figure S7A, Supporting Information), and the quantitative analysis also confirmed the formation of abundant vessels in FCB group (Figure S7B, Supporting Information). These results indicated that FCB hydrogel enhanced wound healing by promoting the thickness of granulation tissue, collagen deposition, and angiogenesis. The antibacterial EPL polypeptide and BGN should be responsible for the good performance of wound healing. Previous studies showed that antibacterial EPLbased polypeptide could significantly promote the formation of collagen tissue and angiogenesis, and the BGN could enhance the formation of blood vessel through activating the hypoxia inducible factor. ${ }^{[8,38,39,52]}$ Thus, the FCB hydrogel demonstrated the excellent wound healing performance. These results further indicated that FCB hydrogel was probably a versatile biomaterial for treating infection, cancer, and tissue injury.

As compared to previous multifunctional biomedical hydrogel, the as-prepared FCB hydrogel possesses several 

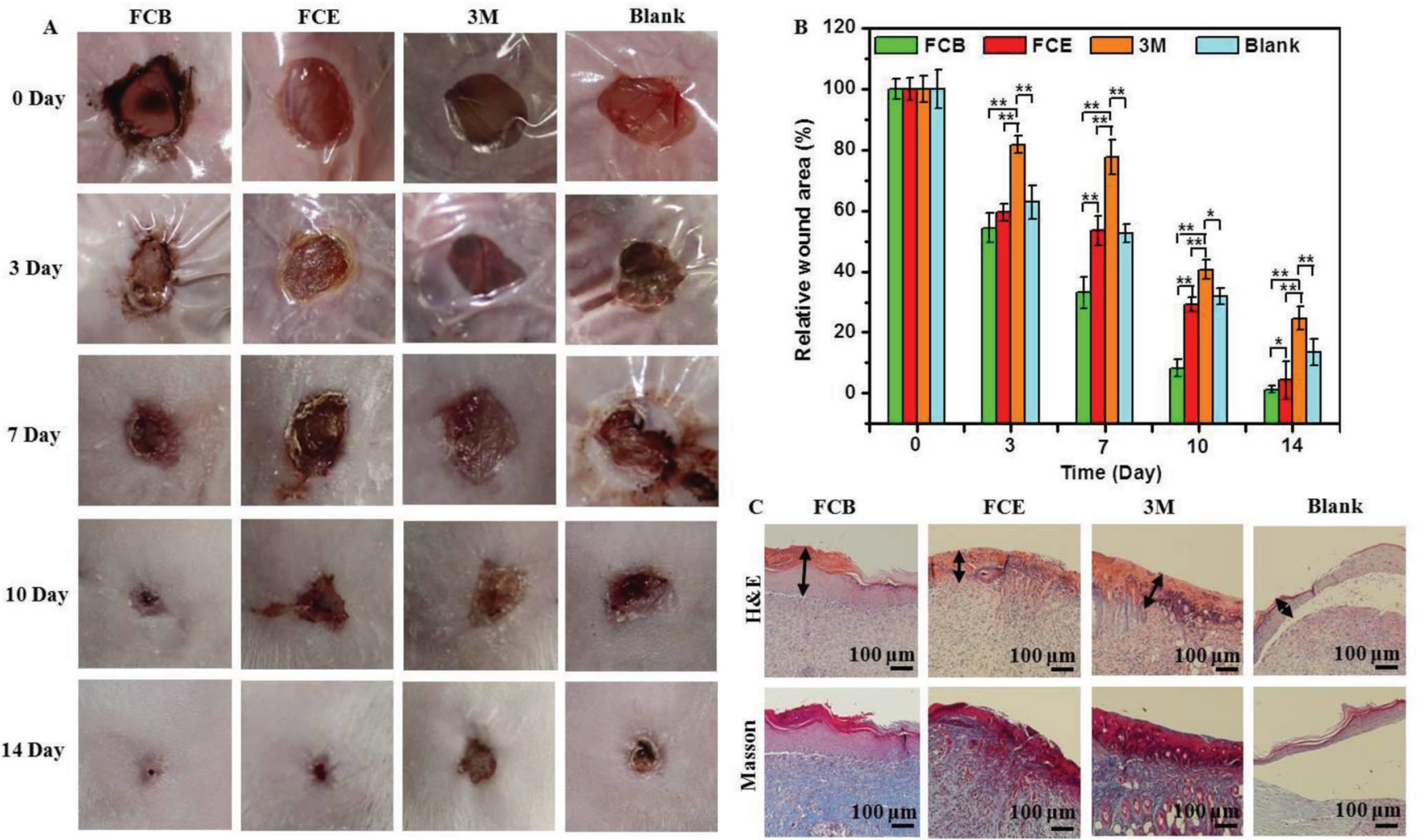

Figure 7. In vivo wound healing in mice. A) Representative skin wound photographs on days $0,3,7$, 10, and 14 and B) wound closure rates of the FCB, FCE, and controls ( $* p<0.05$, ** $P<0.01$, *** $P<0.001$ ); and C) H\&E and Masson's trichrome staining of wounds after $14 \mathrm{~d}$ postsurgery treated with FCB hydrogels and controls, fibrin glue. The black arrows show the granulation layers in wound beds. Scale bar is $100 \mu \mathrm{m}$.

advantages in wound healing. First, FCB hydrogel demonstrated the intrinsical multifunctional capacities including treating multidrug-resistant bacterial infection, inhibiting tumor growth, and enhancing wound healing. These three biomedical functions of FCB hydrogel were few reported for previous biodegradable hydrogels, although the wound healing/anti-infection/antitumor after tumor surgery were rather necessary in helping the recovery of patients. Additionally, the components in FCB hydrogel including EPL, F127, and BGN have shown high biosafety, and the synthesis is facile and suitable for largescale production. Compared with other antibacterial agents including metal ions and polymer, as a natural polypeptide, EPL has the high biocompatibility and excellent broad-spectrum activity including multidrug-resistant bacteria. ${ }^{[8,54,59,60]}$ EPL-based polymers also demonstrated promising applications in tissue regeneration and gene therapy. ${ }^{[8,59]}$ This study provided a facile strategy to construct a bioactive polypeptide-based hydrogel with multifunctional properties for integrated disease therapy-tissue regeneration.

\section{Conclusions}

In summary, a self-healing, injectable, and antibacterial bioactive polypeptide-based nanocomposites hydrogel was developed for anti-infection, skin cancer therapy, and skin repair in vitro and in vivo. FCB hydrogel showed good self-healing and shearthinning behavior with good injectable capacity. FCB hydrogel possessed rapid photothermal performance with effectively killing cancer cells in vitro with negligible cytotoxicity. Additionally, FCB hydrogel demonstrated high antibacterial capability toward E. coli, S. aureus, and MRSA in vitro and in vivo. Importantly, FCB hydrogel could significantly suppress the tumor growth and ablate tumor in vivo through efficient photothermal response. FCB hydrogel could also effectively promote the wound healing in vivo via stimulating the formation of collagen and angiogenesis. This work demonstrates that FCB hydrogel is a promising candidate for anti-infection, tumor therapy, and wound healing. Additionally, the strategy described in this work could be used to prepare other novel hydrogels for multifunctional cancer therapy and tissue regeneration.

\section{Experimental Section}

Fabrication and Characterizations of FCB Hydrogel: Dibenzaldehy determinated F127 (F127-Phe-CHO) and F127-EPL (FEPL) copolymer was prepared in a similar method as described previously, as shown in Supporting Information. ${ }^{[61,62]}$ The FCB hydrogel was prepared through a facile mixing method. Briefly, 20 wt\% F127-Phe-CHO aqueous solution, 40 wt\% FEPL aqueous solution and 5 wt\% BGN@PDA solution aqueous were prepared, respectively. Then, $80 \mu \mathrm{L}$ BGN@PDA solution (0.004 g, $5 \mathrm{wt} \%)$ and $70 \mu \mathrm{L}$ F127-Phe-CHO (0.014 g, 20 wt\%) solution were added to $250 \mu \mathrm{L}$ FEPL solution $(0.1 \mathrm{~g}, 40 \mathrm{wt} \%)$ at ice. After vortexing, the mixture was kept at $37^{\circ} \mathrm{C}$ to form hydrogel. The final volume percentage of FEPL and BGN@PDA were kept 25 and 1 wt\%, respectively. Similarly, the hydrogel using $80 \mu \mathrm{L} \mathrm{H}_{2} \mathrm{O}$ instead of BGN@ PDA was prepared by the same procedure and used as control, denoted 
as FCE (F: FEPL; C: F127-Phe-CHO; E: without BGN@PDA). The characterizations procedures of hydrogel were presented in Supporting Information.

Rheological Mechanical Properties Evaluations: The rheological property (storage $G^{\prime}$ and loss modulus $G^{\prime \prime}$ ) of hydrogels were determined by a TA rheometer (DHR-2, USA). The hydrogels were placed between the parallel plates of $20 \mathrm{~nm}$ diameter and with a gap of $1000 \mu \mathrm{m}$. The temperature effect of various hydrogels in the range of $10-37.5^{\circ} \mathrm{C}$ was evaluated by keeping the strain and frequency constant at $1 \%$ and $1 \mathrm{~Hz}$, respectively. The $G^{\prime}$ and $G^{\prime \prime}$ of hydrogels at $25^{\circ} \mathrm{C}$ or $37{ }^{\circ} \mathrm{C}$ were also recorded. The shear-thinning analysis was carried out through changing the strain (from $1 \%$ to $1000 \%$ with $100 \mathrm{~s}$ interval) for three cycles. The time sweeps were used to indicate the change of $G^{\prime}$ and $G^{\prime \prime}$ were recorded by time sweeps with fixed temperature $\left(25^{\circ} \mathrm{C}\right)$ and frequency $(1 \mathrm{~Hz})$.

Multifunctional Performance Evaluations: The self-healing behavior for hydrogels was measured by macroscopic self-healing experiment. Briefly, the FCB hydrogel was made into 24-well plates $(15 \mathrm{~mm}$ diameter, $3 \mathrm{~mm}$ thickness) with a cavity ( $4 \mathrm{~mm}$ diameter, $3 \mathrm{~mm}$ thickness) and placed for $6-12 \mathrm{~h}\left(25^{\circ} \mathrm{C}\right)$. The self-healing behavior of FCB hydrogel was taken by digital photographs. The followed procedure was used to determine the injectable ability of FCB hydrogel. $400 \mu \mathrm{L}$ FCB hydrogel was prepared in a $1 \mathrm{~mL}$ syringe as described above. The gel was extruded through syringe without clogging, once the solution turned into gel state.

The photothermal effects of the FCB hydrogel, FCE, and $\mathrm{H}_{2} \mathrm{O}$ were determined in a 24-well culture plate upon irradiation by a laser $(808 \mathrm{~nm}$, $1.41 \mathrm{~W} \cdot \mathrm{cm}^{-2}, 10 \mathrm{~min}$ ). FCB without irradiation was used as control. The temperature growth curves and thermal imaging was analyzed through a machine (Fluke VT04A Visual IR Thermometer) in real time.

In Vitro Photothermal Therapy and Cytotoxicity Assay: A375 cells were first cultured under a standard incubation condition $(8000$ per well, DMEM with $10 \% \mathrm{FBS}$ ). After $24 \mathrm{~h}$, the culture solution was changed to be $100 \mu \mathrm{L}$ fresh medium with FCB hydrogel $\left(8 \mu \mathrm{L}, 0.2 \mu \mathrm{g} \mu \mathrm{L}^{-1} \mathrm{FEPL}\right.$, $0.032 \mu \mathrm{g} \mu \mathrm{L}^{-1} \mathrm{~F} 127$-Phe-CHO, $0.08 \mu \mathrm{g} \mu \mathrm{L}^{-1}$ BGN@PDA). The cells were irradiated by an NIR laser for $10 \mathrm{~min}\left(808 \mathrm{~nm}, 1.41 \mathrm{~W} \cdot \mathrm{cm}^{-2}\right)$. As controls, cells with FCE and blank were treated with or without laser. The medium was removed after $24 \mathrm{~h}$ and the cell was washed with PBS for one time. Then, the cells activity was measured through a live-dead kit based on the manufacture's protocol and observed by fluorescence microscope (IX53, Olympus, Japan). As the cell viability on laser treatment, FCB hydrogel treated cells were incubated for $24 \mathrm{~h}$ after NIR illumination $\left(808 \mathrm{~nm}, 1.41 \mathrm{~W} \cdot \mathrm{cm}^{-2}\right.$ for various times). Cells were grown without FCB or with FCB under the same conditions with or without irradiation were used as controls. The cell viability was quantified by Alamar Blue kit as described previously. ${ }^{[53]}$ To investigate the cytotoxicity of FCB hydrogel and each component, $\mathrm{A} 375$ and $\mathrm{C} 2 \mathrm{C} 12$ cells was employed and the experimental procedure was the same as those described above.

Antibacterial Performance Determination: The antibacterial performance of various samples was tested through several representative bacterials including S. aureus (Gram-positive), methicillinresistant Staphylococcus aureus (MRSA), and E. coli (Gram-negative) as reported previously. ${ }^{[63]}$ The in vivo antibacterial test was performed on a MRSA-infected mouse model. The detailed procedure was shown in Supporting Information.

In Vivo Photothermal Tumor Therapy: The subcutaneous melanoma tumor model was employed to analyze the in vivo photothermal capacity for cancer therapy. The detailed procedure for tumor building and photothermal treatment with hydrogels was exhibited in Supporting Information. After PTT for $18 \mathrm{~d}$, the mice were sacrificed and the tumor tissues were removed. The obtained tumors were captured and weighed. To analyze the tissue toxicity, the tumor was fixed by paraformaldehyde in $(4 \% \mathrm{v} / \mathrm{v})$, evaluated using hematoxylin-eosin (H\&E) and TUNEL analysis. All sections were observed using a light microscope (BX53, Olympus, Japan).

In Vivo Wound Healing: The wound healing test of FCB hydrogel in vivo was carried out by a full-thickness cutaneous wound model. Briefly, the female mice (four-week old, 25-30 g) were anesthetized, then a rounded full thickness wound (diameter: $10 \mathrm{~mm}$ ) was created on the back after shaved. The mice were randomized into four groups $(n=5)$ as follows: a control group (Blank), FCB group, FCE group and
Tegaderm $^{3 \mathrm{M}}$ group. Then, the wounds were fitted with an FCB or FCE hydrogel $(50 \mu \mathrm{L})$ before coated with hollow Tegaderm. ${ }^{3 \mathrm{M}}$ The mice did not fitted with hydrogel and shielded with or without Tegaderm were used as controls. All animal experiments procedures were performed according to the protocols approved by the animal care committee of Xi'an Jiaotong University.

Digital photographs of wounds were taken on days $0,3,7,10$, and 14 , and the wound area was determined by Image-J. The wound size was evaluated as follows: relative wound area $=S_{t} / S_{0} \times 100 \%$, where $S_{0}$ and $S_{t}$ were the wound area on day 0 and on day $t(t=3,7,10$, and 14), respectively. On $3,7,10$, and $14 \mathrm{~d}$, specimens of all samples were harvested and stained with $\mathrm{H} \& \mathrm{E}$ and Masson's trichrome based on the manufacture's protocol, respectively. VEGF antibodies were used as the markers of angiogenesis in the wound beds in immunohistochemical staining. Thereafter, the sections were observed by a light microscope (BX53, Olympus, Japan).

\section{Supporting Information}

Supporting Information is available from the Wiley Online Library or from the author.

\section{Acknowledgements}

This work was supported by National Natural Science Foundation of China (Grant No. 51872224), Key Laboratory of Shaanxi Province for Craniofacial Precision Medicine Research, College of Stomatology, Xi'an Jiaotong University (Grant No. 2018LHM-KFKT004), and the Joint Funds of the National Natural Science Foundation of China (Grant No. U1501245).

\section{Conflict of Interest}

The authors declare no conflict of interest.

\section{Keywords}

bioactive nanosystems, multifunctional biomaterials, tissue engineering, tumor therapy, wound healing

Received: September 30, 2018

Revised: March 11, 2019

Published online: March 28, 2019

[1] D. J. Tobin. Chem. Soc. Rev. 2006, 35, 52

[2] M. C. F. Simões, J. J. S. Sousa, A. A. C. C. Pais, Cancer Lett. 2015, $357,8$.

[3] K. D. Miller, R. L. Siegel, C. C. Lin, A. B. Mariotto, J. L. Kramer J. H. Rowland, K. D. Stein, R. Alteri, A. Jemal, Ca-Cancer J. Clin. 2016, 66, 271.

[4] V. S. C. Pua, S. Huilgol, D. Hill, Australas. J. Dermatol. 2009, 50, 171.

[5] M. Wang, Y. Guo, Y. Xue, W. Niu, M. Chen, P. X. Ma, B. Lei, Biomaterials 2019, 199, 10.

[6] A. Stratigos, C. Garbe, C. Lebbe, J. Malvehy, V. del Marmol, H. Pehamberger, K. Peris, J. C. Becker, I. Zalaudek, P. Saiag, M. R. Middleton, L. Bastholt, A. Testori, J. J. Grob, EDF, EADO, EORTC, Eur. J. Cancer 2015, 51, 1989.

[7] M. Wu, Y. Xue, N. Li, H. Zhao, B. Lei, M. Wang, J. Wang, M. Luo, C. Zhang, Y. Du, C. Yan, Angew. Chem., Int. Ed. 2019, https://doi. org/10.1002/ange.201812972. 
[8] Y. Xi, J. Ge, Y. Guo, B. Lei, P. X. Ma, ACS Nano 2018, 12, 10772.

[9] Y. Li, N. Li, J. Ge, Y. Xue, W. Niu, M. Chen, Y. Du, P. X. Ma, B. Lei, Biomaterials 2019, 201, 68.

[10] W. Tao, X. Ji, X. Xu, M. A. Islam, Z. Li, S. Chen, P. E. Saw, H. Zhang, Z. Bharwani, Z. Guo, J. Shi, O. C. Farokhzad, Angew. Chem., Int. Ed. 2017, 56, 11896.

[11] Q. Sun, Q. You, X. Pang, X. Tan, J. Wang, L. Liu, F. Guo, F. Tan, N. Li, Biomaterials 2017, 122, 188.

[12] M. Abbas, Q. Zou, S. Li, X. Yan, Adv. Mater. 2017, 29, 1605021.

[13] J. Li, J. Rao, K. Pu, Biomaterials 2018, 155, 217.

[14] M. Aioub, S. R. Panikkanvalappi, M. A. El-Sayed, ACS Nano 2017, 11, 579.

[15] X. Song, Q. Chen, Z. Liu, Nano Res. 2015, 8, 340.

[16] Y.-W. Chen, Y.-L. Su, S.-H. Hu, S.-Y. Chen, Adv. Drug Delivery Rev. 2016, 105, 190.

[17] Y. L. Liu, K. L. Ai, J. H. Liu, M. Deng, Y. Y. He, L. H. Lu, Adv. Mater. 2013, 25, 1353.

[18] L.-S. Lin, Z.-X. Cong, J.-B. Cao, K.-M. Ke, Q.-L. Peng, J. Gao, H.-H. Yang, G. Liu, X. Chen, ACS Nano 2014, 8, 3876.

[19] W. Chen, Y. Wang, M. Qin, X. Zhang, Z. Zhang, X. Sun, Z. Gu, ACS Nano 2018, 12, 5995.

[20] D. Zhang, M. Wu, Y. Zeng, L. Wu, Q. Wang, X. Han, X. Liu, J. Liu, ACS Appl. Mater. Interfaces 2015, 7, 8176.

[21] G. Pan, S. Sun, W. Zhang, R. Zhao, W. Cui, F. He, L. Huang, S.-H. Lee, K. J. Shea, Q. Shi, H. Yang, J. Am. Chem. Soc. 2016, 138, 15078.

[22] L. Han, X. Lu, M. Wang, D. Gan, W. Deng, K. Wang, L. Fang, K. Liu, C. W. Chan, Y. Tang, L.-T. Weng, H. Yuan, Small 2017, 13, 1601916.

[23] L. Han, X. Lu, K. Liu, K. Wang, L. Fang, L.-T. Weng, H. Zhang, Y. Tang, F. Ren, C. Zhao, G. Sun, R. Liang, Z. Li, ACS Nano 2017, 11, 2561.

[24] D. Gan, T. Xu, W. Xing, X. Ge, L. Fang, K. Wang, F. Ren, X. Lu, Adv. Funct. Mater. 2019, 29. 1805964.

[25] F. Zhao, B. Lei, X. Li, Y. Mo, R. Wang, D. Chen, X. Chen, Biomaterials 2018, 178, 36.

[26] Y. Xue, Y. Du, J. Yan, Z. Liu, P. X. Ma, X. Chen, B. Lei, J. Mater. Chem. B 2015, 3, 3831.

[27] V. Miguez-Pacheco, L. L. Hench, A. R. Boccaccini, Acta Biomater. $2015,13,1$.

[28] Y. Guo, Y. Xue, W. Niu, M. Chen, M. Wang, P. X. Ma, B. Lei, Part. Part. Syst. Charact. 2018, 35. 1800087.

[29] Y. Du, M. Yu, J. Ge, P. X. Ma, X. Chen, B. Lei, Adv. Funct. Mater. 2015, 25, 5016.

[30] J. R. Jones, Acta Biomater. 2013, 9, 4457.

[31] F. Zhao, W. Xie, W. Zhang, X. Fu, W. Gao, B. Lei, X. Chen, Adv. Healthcare Mater. 2018, 7, 1800361

[32] Y. Li, Y. Guo, W. Niu, M. Chen, Y. Xue, J. Ge, P. X. Ma, B. Lei, ACS Appl. Mater. Interfaces 2018, 10, 17722.

[33] M. Yu, Y. Xue, P. X. Ma, C. Mao, B. Lei, ACS Appl. Mater. Interfaces 2017, 9, 8460.

[34] Y. Xue, Z. Zhang, W. Niu, M. Chen, M. Wang, Y. Guo, C. Mao, C. Lin, B. Lei, Part. Part. Syst. Charact. 2019, 1800507.
[35] Y. Xue, Y. Guo, M. Yu, M. Wang, P. X. Ma, B. Lei, Adv. Healthcare Mater. 2017, 6, 1700630

[36] C. Wu, J. Chang, J. Controlled Release 2014, 193, 282.

[37] F. Li, M. Wang, G. Pi, B. Lei, J. Biomed. Nanotechnol. 2018, 14, 756.

[38] W. Gao, W. Jin, Y. Li, L. Wan, C. Wang, C. Lin, X. Chen, B. Lei, C. Mao, J. Mater. Chem. B 2017, 5, 7285.

[39] C. Wang, Q. Wang, W. Gao, Z. Zhang, Y. Lou, H. Jin, X. Chen, B. Lei, H. Xu, C. Mao, Acta Biomater. 2018, 69, 156.

[40] B. Lei, B. Guo, K. J. Rambhia, P. X. Ma, Front. Med. 2018, https:// doi.org/10.1007/s11684-018-0664-6.

[41] S. Kargozar, M. Mozafari, R. G. Hill, P. B. Milan, M. T. Joghataei, S. Hamzehlou, F. Baino, Mater. Today: Proc. 2018, 5, 15532.

[42] R. Xing, K. Liu, T. Jiao, N. Zhang, K. Ma, R. Zhang, Q. Zou, G. Ma, X. Yan, Adv. Mater. 2016, 28, 3669.

[43] X. Bai, M. Gao, S. Syed, J. Zhuang, X. Xu, X.-Q. Zhang, Bioact Mater. 2018, 3, 401.

[44] J. Fu, M. in het Panhuis, J. Mater. Chem. B 2019, https://doi. org/10.1039/C9TB90023C.

[45] R. Li, J. Xu, D. S. H. Wong, J. Li, P. Zhao, L. Bian, Biomaterials 2017, $145,33$.

[46] K. Zhang, Q. Feng, J. Xu, X. Xu, F. Tian, K. W. K. Yeung, L. Bian, Adv. Funct. Mater. 2017, 27, 1701642.

[47] K. Zhang, S. Lin, Q. Feng, C. Dong, Y. Yang, G. Li, L. Bian, Acta Biomater. 2017, 64, 389

[48] V. Pertici, C. Pin-Barre, C. Rivera, C. Pellegrino, J. Laurin, D. Gigmes, T. Trimaille, Biomacromolecules 2019, 20, 149.

[49] Y. Wu, H. Wang, F. Gao, Z. Xu, F. Dai, W. Liu, Adv. Funct. Mater. 2018, 28, 1801000.

[50] C. Wang, M. Wang, T. Xu, X. Zhang, C. Lin, W. Gao, H. Xu, B. Lei, C. Mao, Theranostics 2019, 9, 65 .

[51] J. Ge, K. Liu, W. Niu, M. Chen, M. Wang, Y. Xue, C. Gao, P. X. Ma, B. Lei, Biomaterials 2018, 175, 19.

[52] B. Hu, Y. Shen, J. Adamcik, P. Fischer, M. Schneider, M. J. Loessner, R. Mezzenga, ACS Nano 2018, 12, 3385.

[53] L. Zhou, X. Qu, Y. Guo, M. Wang, B. Lei, P. X. Ma, ACS Appl. Mater. Interfaces 2018, 10, 4471.

[54] L. Zhou, Y. Xi, M. Yu, M. Wang, Y. Guo, P. Li, P. X. Ma, B. Lei, Acta Biomater. 2017, 58, 90.

[55] L. Han, K. Liu, M. Wang, K. Wang, L. Fang, H. Chen, J. Zhou, X. Lu, Adv. Funct. Mater. 2018, 28, 1704195.

[56] L. Klouda, Eur. J. Pharm. Biopharm. 2015, 97, 338.

[57] K.-Q. Wang, S.-Z. Luo, X.-Y. Zhong, J. Cai, S.-T. Jiang, Z. Zheng, Food Chem. 2017, 214, 393.

[58] S. L. Percival, K. E. Hill, D. W. Williams, S. J. Hooper, D. W. Thomas, J. W. Costerton, Wound Repair Regener. 2012, 20, 647.

[59] Y. Du, J. Ge, Y. Li, P. X. Ma, B. Lei, Biomaterials 2018, 157, 40.

[60] L. Zhou, Y. Xi, M. Chen, W. Niu, M. Wang, P. X. Ma, B. Lei, Nanoscale 2018, 10, 17304

[61] T. Liu, X. Zhang, B. Ke, Y. Wang, X. Wu, G. Jiang, T. Wu, G. Nie, Mater. Sci. Eng., C 2016, 61, 269.

[62] X. Yang, G. Liu, L. Peng, J. Guo, L. Tao, J. Yuan, C. Chang, Y. Wei, L. Zhang, Adv. Funct. Mater. 2017, 27. 1703174.

[63] S. R. Deka, A. K. Sharma, P. Kumar, Curr. Top. Med. Chem. 2015, 15, 1179. 